\title{
Comprehensive Discussion on Editing and Publishing Network
}

\author{
Zhan Xinrui \\ Weinan Normal University, Newspaper Community, Weinan, Shaanxi, 714099
}

Keywords: Editing and Publishing, Modernizing, Networking, Sharing, Individualization

\begin{abstract}
The network of editing and publishing is the characteristic of editing and publishing in the era of network. It is the specific application of large data and Internet technology in the field of editing and publishing. The editing and publishing of network to the modernization of society as the realistic foundation, the subject consciousness of the editing and publishing network as the ideological basis for the development of information technology and network technology as the foundation of the technology; the main contents include editing network publishing subject consciousness of editors, network publishing object processing methods, edit publications show network carrier form. Editing and publishing network are shared, flexibility, diversity and individuality, which is a world without end.
\end{abstract}

\section{Introduction}

With the rapid development of computer information technology and the Internet, McLuhan predicted the "global village" has been realized over 60 years ago. The world is undergoing profound changes due to the Internet. People's work, life, study and even way of thinking are gradually networked. Editing and publishing as a popular form of paper media in the process of the birth of occupation, has completed the "lead and fire" to "light and electricity" gorgeous, and is swept toward "number and network form, editing and publishing network has become the development direction of editing and publishing industry can not avoid. The development of the industry will attract the attention and research of relevant scholars [1-2]. However, for the current research situation, there are relatively few researches on the editing and publishing networking in academic circles, and no more theoretical papers and works on editing and publishing networking. This paper tries to define the basic concept of network publishing from editing, editing and publishing based on the network of mining, a theoretical discussion and the basic content of the editing and publishing of network and the typical characteristics and so on, in order to provide a theoretical premise for the editing and publishing of the basic theory research network.

\section{The Concept of Editing and Publishing Network}

Networking means relying on information and communication technology and computer network technology to connect remote electronic terminals to certain network protocols, forming information superhighway and resource sharing platform, so as to achieve the purpose of resource sharing for all users. The modernization of editing and publishing is a dynamic process that editors and publishers constantly apply the most advanced science and technology and management concepts to the editing and publishing field, act on the object of editing and publishing, and constantly improve the editing and publishing technology level. In the process of editing and publishing from tradition to modernization, the subject, object and carrier of editing and publishing have been branded with network. The network of editing and publishing is a concrete manifestation of the modernization of editing and publishing in the network era [3]. Therefore, the concept of editing and publishing network were defined as follows: the editing and publishing network refers to the main body of the editing and publishing network thinking consciousness as the guide, the editing and publishing of the object of soliciting manuscripts, editing, review and assessment of a series of process in the form of networking, and the realization of a new editor edit the publication publishing form vector of flexibility, individuality and autonomy. In order to clarify the concept of 
editing and publishing, it is necessary to distinguish between the following two groups of concepts:

\subsection{Editing and publishing network and network publishing}

"Network publishing, as the name suggests, is the traditional text information stored in digital form in a variety of computer storage medium, and then spread through the Internet, the last people to read the text information through various electronic products similar to the PC terminal." The main features of network publishing, digital publishing is the product of current network products, electronic transactions, the main emphasis on the publications of the carrier from paper to digital transformation and publication circulation spread from the traditional physical distribution to digital communication with the network, it emphasizes the digital alternative to paper. The networking of editing and publishing is a process of omnidirectional networking from editing, publishing subject, object and carrier. It emphasizes the sharing of publishing resources, the digitalization of publishing process and the individuation of publication form. The network of editing and publishing is not a substitute for the paper, but a complementarity [4].

\subsection{Editing and publishing network and digital publishing}

Digital publishing is a new publishing form that can be transformed into binary code by computer digital technology and stored on mobile hard disk, disc and other media. Digital publishing has the characteristics of easy to copy and portable. Digital publishing emphasizes the content, production mode, action process, transmission carrier, and digitization of reading consumption. Digital publishing and editing and publishing networking emphasize digitalization, while digital publishing mainly emphasizes the substitution of technology in editing and publishing process, and emphasizes the paperless form of publication. Editing and publishing network is not only improving the traditional publishing form at the technical level, but also emphasizing the change of editorial and publishing subjects in the level of thinking consciousness, and highlighting diversity in the form of publication. Digital publishing is a specific form of publishing. Editing and publishing networking is the goal pursued by editors and publishers. It is a process of continuous improvement and endless.

\section{The Content of Editing and Publishing Network}

Editing and publishing network is a subject to editing and publishing from the object to the full range of network carrier, it mainly includes editing network publishing subject consciousness, editing and publishing object processing method of network carrier and edit publications present form of the network in three aspects.

\subsection{Network of thinking consciousness of the subject of editing and publishing}

As we have discussed before, editing and publishing network should be based on the networking of editing and publishing consciousness. The way of editing and publishing is decided by the thinking consciousness of editors and publishers. First, effective sharing of editing and publishing network subject consciousness requires editing and publishing subject can achieve information and maximize the utilization, which requires the subject to editorial consciousness, is the requirement of editing and publishing body has "cultural view", a "media medium" concept, with "culture" literacy, master "media skills. For example, the press and publication has its own relatively strong publishing and research direction, when the editors received is not consistent with their field release, should be able to recommend sharing manuscript to the corresponding publishing unit, the sharing of resources can maximize the publishing unit strengths, and make the thoughts and ideas spread maximization, is to maximize the use of resources [5-6]. The network of the thinking consciousness of the subject of editing and publishing requires the editor and publication subject to pay attention to consumer's consumption experience and set up the consciousness of service. An important impact of human entry into the Internet age on information dissemination is to change the passive position of the audience in the process of information acceptance, and the audiences turn from the casual spectator and the passive receiver to the active choice and active participant. Therefore, as an editor 
of information producers, we should take the audience's consumption experience as the starting point of information production, and we should use various ways to collect the audience's feedback and try to satisfy the audience's consumption needs. In the traditional three trial five school editing system, editing and proofreading and issuing are different links. The main body of editing and publishing is not responsible to the audience, but responsible for the system, so long as the corresponding editing and proofreading process can be completed. But in the networked environment, editors are directly faced with consumers. Editors need to be accountable to consumers. Editors pursue quality and focus on consumers' experience and feedback. Therefore, the network of the thinking consciousness of the editor and publishing subject requires the editor and publication subject to change from the dominant to the service type and set up the service consciousness.

\subsection{The networking of the processing mode of the object of editing and publishing}

"From the author's contribution to the editorial department, the object of every part of the editorial department, until the last publication, we call it the object of editing and publishing. The starting point is to edit the editing and publishing work to the author or authors to directional manuscripts editing and publishing sector delivery articles, or whether it is submitted manuscripts in the form of networking is very important, it will directly affect the subsequent processing of manuscripts. The editorial department of Journal of Weinan Teachers University has successfully applied for the Ministry of education's "Sima Qian and" Shiji "research, and the publication changed to half monthly [7]. The circulation of manuscripts is very large in a long time. In addition to the relevant section of the manuscript, need to download, registration number of all my published articles on Social Sciences and related to editing, every day there are at least fifty or sixty articles need to download the registration problem, and some authors do not repeat the submission or timing of natural science and social science papers wrong etc.. It takes a lot of time to process these manuscripts every day, and from time to time, repeated registration or missed registration occurs. If a leave or other causes are delayed, the manuscript will not arrive at the editor's hand, which will affect the timeliness of the manuscript processing. In view of this reason, our editorial department was launched in March 2016 by Chinese HowNet to provide "periodical collaborative editing system", the author at the time of submission must enter my print online submission system, and the real name registration, complete manuscript Abstract keywords English translation, author information, contact information and a series of information, and the need for accurate selection of manuscript categories after uploading articles attachments [8]. When the editors of related columns $\log$ on to the contributor system with their own accounts, they can see the author's contribution for the first time, and have detailed author information and contact information. Authors and editors and reviewers can achieve real-time, unified registration number, and save the process, but also avoid due to the carelessness of the author, forget information in the mail submission and make editing cannot contact the author, and the system of investment platform for manuscript submission through automatically "academic misconduct detection". At the same time, the author can pay attention to the processing of the manuscript at any time, and can modify the manuscript at any time through the feedback of the editor. There are other professional editing systems (such as three manuscript editing system) can realize the authors, editors, reviewers on communication, editing preliminary views, expert opinions, the author of the review, modify the manuscript published, a series of information can be displayed in the editing system, as far as possible, fair and open treat every manuscript, the maximum shortening processing time. "At the same time, the use of periodical network management platform can save a lot of business expenses, including newsletters between editorial department, experts, authors and readers, postal charges, communication charges generated by telephone connection, print fees produced by authors, proofreading manuscripts, etc."

In the process of editing and processing manuscripts, the use of a series of large network databases can also be used to assist in editing and processing. Analysis of the "dark horse Checker" covering the social and natural sciences in various fields of the Chinese corpus of 100 billion words, with 79 professional thesaurus, 48 million professional vocabulary, 4 million 500 thousand errors 
were compared to the core library online editing the manuscript, check the accuracy is very high, can well make up for proofreading the knowledge in the blind spots and omissions [9]. SUCCHI "Note First reference Aided Editing System" according to the authoritative database automatically add references in missing data can be modified, author, title, year volume of page data in the literature, also can modify small letters, abbreviations, punctuation, author name order, check references, reference the citation is continuous and a series of problems. The use of these large network databases can greatly reduce the workload of editing and processing, and make the information more accurate and reliable, shorten the editing time and improve the quality of editing and proofreading. With the improvement of big data and cloud computing technology, more networked editing, processing and proofreading systems will be applied to the daily work of editing and publishing, changing the working mode of editing and publishing subjects, and making the editing and publishing objects more networked.

\subsection{The networking of the presentation form of the editors' publication carrier}

Mr. Zhang Jiyu in "the modernization of editing and publishing a paper on" that modern edit publications should be consistent with the novelty, form and content of the characteristics of the carrier, loved by comprehensive and exquisite graphic design etc.. In the development process of editing and publishing networking, not only adheres to editorial guidelines for editing publications, but also requires the freedom and flexibility of editing publications, the variety of carriers and the individualization of binding design. Edit the publication the present form of the network carrier is not required to edit the publication from the physical form of traditional fully into the network as the carrier of the digital form, because we have no power to deprive the traditional paper publications give readers the unique experience of reading, the reading experience is the other digital audio-visual media can not be given. The publication form of the publication carrier is networked. First, the publication that the editorial department sells to the audience is not a one-time stereotype product, but a lifelong service. With the rapid development of mobile media and wireless network technology, more and more publications are selected in the form of networking publishing, network publications editor does not refer to the digital transformation of publications on paper product content, but require the publication of products and customer end is a network sharing platform. Publishing houses should update contents at any time to ensure that users get the latest content, that is, online product services. Once consumers purchase products, they will also buy lifelong services.

The networking of the presentation forms of the editors' publications also requires the editing of the publications to be personalized and produced on demand. Personalized and on-demand production is not difficult for the easily replicated digital products, but it will be challenging for publications that will eventually be presented in paper form. We try to depict the future such a scenario, the commercial press will publish a book, but he did not give the author provides at least how many printed books, even printing factory did not print the book, but editing a manuscript published in molding system. A thousand of miles away from the readers to see the book in a terminal system similar to the Bank Street automatic deposit machine, so he chose the system default font typesetting, love their name, and they just uploaded a shot of the family portrait photographs as book cover elements. The personalized color is selected and then clicking the print button lightly. After a few minutes, a self-designed publication with its own unique style is presented. Moreover, the system can record readers' information of the book in detail and share it with all publishers, authors and readers. The author can clearly and accurately get the readers of his works. Readers can share their reading comprehension at any time through the mobile terminal. The press also can monitor book sales and distribution between readers, even all over the country readers can also free talk, exchange of views, can also investigate errors and problems in the publication, the author to revise and improve the timely publication, perfect in the author constantly reading experience. This network publishing form will be edited and published in the main editing and proofreading, the author examined the publisher printed, the readers' information feedback and a series of activities "wiped out", truly editing, publishing and reading feedback integration. 


\section{Characteristics of Editing and Publishing Network}

\subsection{Sharing}

Sharing is sharing, which is the right to use or know the right of use or information of an item or information to share with others. In the Internet era, news information is increasing rapidly, entertainment information is rising rapidly, advertising information is spreading everywhere, and technology information is increasing rapidly. The main reason for the audience to feel the explosion of information is information sharing. Sharing is the primary feature of network information. Therefore, the network - based editing and publishing network has the characteristics of sharing. The sharing of editing and publishing is first manifested in the sharing of resources. As mentioned earlier, when the traditional paper publication can also free personalized customized, all publishing departments will share a publishing platform, and share the product output terminal equipment, different publishers only according to their own strengths to produce content, share network, sharing equipment, also share the author, readers sharing. The sharing of editing and publishing is also manifested in the sharing of information [10]. In the editing and publishing of network under the background of the publishing sector is not only the competition, more important is the cooperation, complementary relationship, they shared the audience feedback information, sharing the writer's information sharing, the development of the information industry, each one takes what he needs from each other, mutual benefit and win-win cooperation. At the same time, the audience and the author in this system can also get all kinds of information in a fair and open way to ensure the freedom of the audience and the author's creation.

\subsection{Flexibility}

With the division of labor and the diversification of consumption demand, the location of the audience has shifted from the public to the sub public and then to the minority. Any product can only meet a small number of people's consumption needs, and the needs of the audience are constantly changing. Therefore, in order to continue to meet the consumer demand for information and culture products, it is necessary for the editing and publishing department to have a strong flexibility. First of all, need to edit the publication department at any time according to the needs of the society and the audience to adjust its publishing program, and can fast short-term planning topics according to the needs, with perfect emergency plan and feasible; secondly, editing and processing time of editing and publishing departments to publish content in the guarantee and does not affect the quality of the publications as far as possible to shorten the publication. As far as possible the use of editing and publishing platform to reduce the editing and publishing of objects between editors and authors and publications in the publishing sector and the audience's circulation time, flexible processing and editing process, ensure the timeliness of information products; thirdly, the creation process of editing and publishing in the network also has great flexibility, the author is not completely dominant also, the audience is not completely passive, the audience can also be appropriate to participate in the creative process. From the beginning of topic selection, editors, authors and audiences communicate with each other, plan together and share topics together, and integrate the needs and ideas of the audience into the author's creation.

\subsection{Richness}

The richness of editing and publishing network is mainly reflected in the form of the carrier, the way of presentation, the way of communication and so on. Digital technology to cloud storage and highway network, completely changed the traditional publication single paper carrier form, has formed a paper based on network technology, video and audio books for omnibearing publishing derived. Various publications form is not a simple copy of the same content, but complement each other, and is compatible with each one has his good points, audio-visual, both God shaped various sensory systems in the audience, the audience have hitherto unknown reading and audio-visual experience, and to the audience, referral patterns and forms the order of publication has changed change. For example, in the traditional publishing system, a good novel first after ten years of grinding sword cut the marking, editing audit editing three trial five schools, plus the printing layout, 
transport distribution, promotion and a series of measures is likely to be aware of and buy. After several decades of precipitation and accumulation, it is possible to be moved to the screen. In the context of editing and publishing network, the content creation, propaganda and introduction, screenplay adaptation and film and television photography of the novel can all be carried out at the same time. Some of the more selling novels are published in subsequent volumes in the reader's time and again, and at the same time will be the film investors to adapt to the screen, audiobooks, radio and other means will meet with consumers in a very short period of time. The diversity of publications carrier forms the richness of readers' consumption form, and then can satisfy different levels of audience groups from various ways, expand the influence of works and enhance their communication effects.

\subsection{Personalization}

As previously mentioned, updating, editing and publishing network can be achieved not only exists in digital form of publication of the lifelong service, but also in the near future will achieve print personalized publishing, editing and publishing the service object from group to group to individual, personalized and free. Therefore, the individualized characteristics of the editing and publishing network first refer to the personalization of the form of publication and the way of service. At the same time, when the publishing department can realize information exchange, resource sharing, want to occupy a space for one person in the reader's mind, also need to press planning and production of special, personalized publications, also is to meet the personalized needs of the audience and form their own characteristics, not only in form, but also to have the distinct characteristics in content. In order to meet the individual needs of the audience, it can guide the consumption trend of the audience, stimulate the consumption demand of the audience, and finally realize the personalized customization service.

\section{Conclusion}

The network of editing and publishing is the concrete manifestation of editing and publishing in the Internet age, and is the mainstream mode and development direction of the current editing and publishing industry. Editing and publishing network is a process, not a result, it is based on the Internet technology as the support, emphasizing the development of ideas of editing and publishing subject in terms of thinking consciousness and service idea in line with the thinking of the Internet, convenient, and can realize the form of publication of its work mode diversification, personalized service etc. It will develop along with the Internet technology and the Internet thinking is a world without end.

\section{References}

[1] Zhao L. Application of Network Technology in Sci-tech Journal Editing and Publishing Process[C]// International Conference on Information Engineering for Mechanics and Materials. 2016.

[2] Bolland J. General Writing, Editing and Publishing Considerations[M]// Writing Securities Research: A Best Practice Guide, Second Edition. John Wiley \& Sons, Inc. 2015.

[3] Chung Y. Topics of major current interest in scholarly editing and publishing based on the content analysis of selected journals[J]. 2015, 2(2):59-62.

[4] Gottlieb S M. SYSTEMS AND METHODS FOR CREATING, EDITING AND PUBLISHING RECORDED VIDEOS:, US 9711181 B2[P]. 2017.

[5] Edwards B F R. Ruth Buck and the publication of Edward Ahenakew's Voices of the Plains Cree. Historical Perspectives on Canadian Publishing[J]. La Houille Blanche, 2016:305-307.

[6] Jin J I. Journal editing and publishing practices during the first quarter century since the establishment of the Korean Chemical Society in 1946[J]. Journal of Applied Aquaculture, 2015, 
2(1):3-9.

[7] Vega A M. Bibliometric study and network analysis of the phenomenon of self publishing [C]// International Conference on Technological Ecosystems for Enhancing Multiculturality. ACM, 2015:439-447.

[8] Linder D. English abstracts in open access translation studies journals in Spain (2011-12): Errors in the Writing, Editing and Publishing Chain [J]. Information Resources Management Journal, 2014, 27(3):12-27.

[9] V. Vičić-Hudorović, N. Hudorović, A. Yuri Gasparyan. Do we have tools for quality assurance in science editing and publishing? [J]. European Science Editing, 2014, 40(4):86-87.

[10] Wei X, Liu W, Cong H, et al. Implementation and achievements of project for high quality scientific journals by China Association for Science and Technology(CAST) :a case study of Transactions of the Chinese Society of Agricultural Engineering[J]. Transactions of the Chinese Society of Agricultural Engineering, 2014, 30(17):336-340. 